\title{
Two-Hop Networks with Energy Harvesting: The (Non-)Impact of Buffer Size
}

\author{
Burak Varan Aylin Yener \\ Wireless Communications and Networking Laboratory \\ Electrical Engineering Department \\ The Pennsylvania State University, University Park, PA 16802 \\ varan@psu.edu_yener@ee.psu.edu
}

\begin{abstract}
In this paper, a two-hop channel is considered with energy harvesting transmitter nodes. In particular, the offline throughput maximization problem is solved for a constant power relay, and a relay with one energy arrival, in both cases assuming a finite buffer is available at the relay for temporarily storing data received from the source. The focus is on assessing the impact of this data buffer at the relay on optimal transmission policies. The solution is found indirectly, by first assuming that the relay has an infinite size buffer, and then proving that an optimal policy exists that does not require any data buffer at the relay, thus solving the problem regardless of the data buffer size at the relay. Numerical results demonstrate that the proposed solution performs significantly better than naïve policies, and a constant relay rate limits the average throughput as the peak energy harvest rate for the source increases.
\end{abstract}

Index Terms-Energy harvesting nodes, two-hop channel, relay channel, throughput maximization, finite buffer.

\section{INTRODUCTION}

We consider a two-hop relay network with an energy harvesting source node and a relay that has constant power or a fixed energy budget. The data buffer at the relay is assumed to have a finite capacity which introduces a new constraint on the feasible space of transmission policies and renders the problem of throughput maximization challenging. We attack this problem by relaxing the finitebuffer constraint and showing that this upper bound is achievable when the relay has a buffer of any finite size including zero.

Energy harvesting has been studied in many channel settings in the recent years. References [1] and [2] consider a single-user communication system with energy harvesting and show that a piecewise linear power policy is the optimal offline policy both when the capacity of the battery at the source node is infinite and when it is finite, for transmission completion time minimization and throughput maximization. The extension to fading channels is considered in [3]. Multi-terminal models are studied in [4]-[8] including the multipleaccess channel, broadcast channel, interference channel and the relay channel.

Of particular relevance to this work are references [9]-[12] which study the two-hop channel with energy harvesting nodes. In [9], the solution to the throughput maximization problem is given for a fullduplex relay, and for the half-duplex case the solution is given for one energy arrival at the source. In [10] and [11], the same problem with a half-duplex relay is studied, properties of the optimal policy are derived and the solution with two energy arrivals at the source is given. In [12], a two-hop channel is studied with constraints on the peak relay power and total energy that the relay spends. In all of these works, the relay is assumed to have an infinite size buffer in which it can store data until an opportune time arises to transmit from an energy perspective.

In this paper, we consider a relay with finite buffer with the goal of studying the impact of the buffer size on the optimum policy.

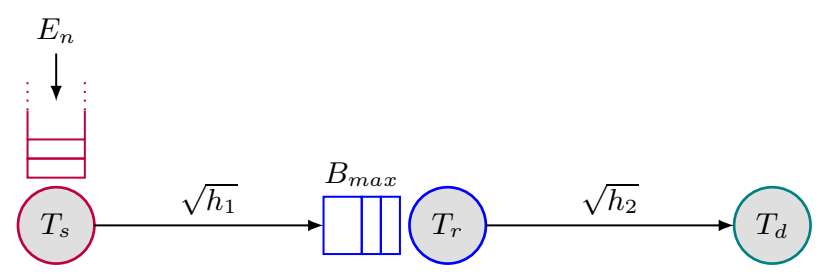

Fig. 1: The two-hop channel with energy harvesting.

As it was observed in references [9]-[11], the half-duplex problem with multiple energy arrivals at the nodes is challenging and remains challenging with the buffer size constraint. We thus consider two special cases for energy availability at the relay. Specifically, we consider either a constant power relay or one that gets a singular energy arrival, i.e., one with a fixed energy budget. The source node is energy harvesting with an arbitrary number of energy arrivals. We address the throughput maximization problem and first find the relaxed solution for an infinite size buffer. Then, we prove that there exits an optimal policy for the relaxed problem that does not require a data buffer at the relay, thereby solving the problem regardless of buffer size.

The remainder of the paper is organized as follows. In Section II, we describe the system model. In Section III, we identify properties of the optimal policy and describe the solutions with constant relay power and single energy arrival at the relay. In Section IV, we present numerical examples to evaluate the performance of the proposed algorithm. We discuss our findings and conclude the paper in Section V.

\section{System Model and Problem Statement}

We consider a Gaussian relay channel consisting of a source node, a relay and a destination node, denoted by $T_{s}, T_{r}$ and $T_{d}$, respectively (Fig. 1). The destination cannot hear the source directly, thus communication is possible only through the relay. Let $h_{1}$ and $h_{2}$ denote the power gain in the first hop, from $T_{s}$ to $T_{r}$, and the second hop, from $T_{r}$ to $T_{d}$, respectively. $h_{1}$ and $h_{2}$ are normalized with respect to the noise variance at the respective receiver such that the Gaussian noise at the relay and the destination has unit variance.

The source $T_{s}$ harvests energy in an intermittent fashion from external sources. The battery at the source is assumed to have an infinite capacity. $E_{n}$ denotes the amount of energy that arrives at the source at the $n$th energy harvest. The energy available in the source's battery before transmission starts is treated as the first arrival and this amount is denoted by $E_{1}$. Communication takes place until a deadline $T$ and $N$ denotes the number of energy arrivals until the deadline. The time duration between the $n$th and $(n+1)$ th energy arrivals is referred to as the $n$th epoch and its length is denoted 
by $l_{n} . s_{n}$ denotes the time that the $n$th energy arrival occurs with $s_{1}=0$ and $s_{n}=\sum_{i=1}^{n-1} l_{i}, n=2,3, \ldots, N$. We also set $s_{N+1}=T$ for convenience. All energy arrivals are assumed to be known noncausally as in references [1]-[12].

For the energy availability at the relay, we consider two cases. In the first case, we assume that the relay can maintain a constant power, $P$, throughout the transmission. In the second case, we consider a relay that receives $E$ units of energy at the beginning of transmission. The relay has a data buffer that it can use to store incoming data that it will forward later. The data buffer can at most store $B_{\max }$ units of data. This introduces a new constraint on the feasible set of transmission policies since any policy that allows more than $B_{\max }$ units of data in the relay buffer at any time should not be employed. The relay in our model is a half-duplex node, so it cannot receive and transmit simultaneously, i.e., $p_{s}(t) p_{r}(t)=0$ where $p_{s}(t)$ and $p_{r}(t)$ denote the instantaneous power values at time $t \in[0, T]$ for the source and the relay, respectively. The instantaneous rates achieved by these power values are $C\left(h_{1} p_{s}(t)\right)$ and $C\left(h_{2} p_{r}(t)\right)$ where $C(x)=$ $\frac{1}{2} \log (1+x)$. The total data transmitted by time $t$ is given by $R_{s}(t)=$ $\int_{0}^{t} C\left(h_{1} p_{s}(\tau)\right) d \tau$ for the source and $R_{r}(t)=\int_{0}^{t} C\left(h_{2} p_{r}(\tau)\right) d \tau$ for the relay. Similarly, the energy spent by time $t$ is given by $E_{s}(t)=$ $\int_{0}^{t} p_{s}(\tau) d \tau$ for the source and $E_{r}(t)=\int_{0}^{t} p_{r}(\tau) d \tau$ for the relay.

Considering all the constraints introduced by the system model (see also [9]-[12]), the throughput maximization problem for a constantpower relay is given by

$$
\begin{aligned}
& \max R_{r}(T) \\
& \text { s.t. } 0 \leq R_{s}(t)-R_{r}(t) \leq B_{\max }, \\
& E_{s}(t) \leq \sum_{n: s_{n}<t} E_{n}, \\
& p_{s}(t) \geq 0, p_{r}(t) \geq 0, p_{s}(t) p_{r}(t)=0, \forall t \in[0, T] .
\end{aligned}
$$

The throughput maximization problem for single energy arrival at the relay is described by adding the constraint $E_{r}(T) \leq E$ to optimization problem (1). Instead of directly solving (1) and its counterpart with the relay of fixed energy budget, we will restate the problems with discrete decision variables and relax the finite-buffer constraint, which will provide upper bounds for the original problems. We will then show that they are achievable regardless of buffer size. In the next section, we restate (1) and identify some properties of the solution of the relaxed problem that will help characterize the solution of (1).

\section{Optimal Policies}

\section{A. Properties of the Optimal Policy for $B_{\max }=\infty$}

Let us relax (1b), i.e., set $B_{\max }=\infty$, and identify the properties of the optimal policy that solves this relaxed problem both with constant relay power and single energy arrival at the relay. Some of these properties have already been proved in [10] for a general energy harvesting two-hop channel which we mention here for completeness. These are: (i) in the optimal policy, the source or the relay should keep its power constant in a single epoch whenever it transmits [10, Lemma 1]; (ii) either the source or the relay should be transmitting at any time during transmission [10, Lemma 3]; (iii) the power levels of the source and the relay should form a nondecreasing sequence [10, Lemma 4]; (iv) the source battery should be empty whenever the source power changes [10, Lemma 5]; and (v) the source and the relay should transmit the same amount data by the deadline $T$ for optimality [10, Lemma 8]. In the two special cases considered in this paper, it is possible to identify additional properties as follows.

Lemma 1: For each feasible policy, there exists an equally well performing feasible policy in which every epoch is composed of two sub-epochs such that the source transmits in the first sub-epoch and the relay transmits in the second one.

Proof: We can merge all time intervals in a single epoch in which the source transmits in a sub-epoch and move this sub-epoch to the beginning of the epoch. During the remainder of the epoch, the relay transmits. Since the amount of energy spent by the source is the same and energy arrives at the beginning of the epoch, (1c) is not violated. The relay also uses the same amount of energy to transmit the same amount of bits, but part of its transmission is delayed without violating feasibility since $B_{\max }=\infty$.

Lemma 1 suggests that we define a new parameter $\Delta_{n}, n=$ $1,2, \ldots, N$ that denotes the fraction of the $n$th epoch that the source transmits. Then, the lengths of the source and relay sub-epochs in the $n$th epoch are given as $l_{n} \Delta_{n}$ and $l_{n}\left(1-\Delta_{n}\right)$, respectively. Clearly, $0 \leq \Delta_{n} \leq 1, n=1,2, \ldots, N$, and if only the source (resp. relay) transmits in the $n$th epoch, then $\Delta_{n}=1$ (resp. 0).

With the properties of the optimal policy from [10] and Lemma 1 at hand, we can restate the relaxed version of (1) as

$$
\begin{aligned}
& \max _{p_{s, n}, p_{r, n}, \Delta_{n}} \sum_{n=1}^{N} l_{n} \frac{1-\Delta_{n}}{2} C\left(\frac{h_{2} p_{r, n}}{1-\Delta_{n}}\right) \\
& \text { s.t. } 0 \leq \sum_{i=1}^{n} l_{i}\left(\frac{\Delta_{i}}{2} C\left(\frac{h_{1} p_{s, i}}{\Delta_{i}}\right)-\frac{1-\Delta_{i}}{2} C\left(\frac{h_{2} p_{r, i}}{1-\Delta_{i}}\right)\right), \\
& \sum_{i=1}^{n} l_{i} p_{s, i} \leq \sum_{i=1}^{n} E_{i}, \\
& p_{s, n}, p_{r, n} \geq 0,0 \leq \Delta_{n} \leq 1, \forall n=1,2, \ldots, N,
\end{aligned}
$$

where $p_{s, n}$ and $p_{r, n}$ denote the average power values of the source and the relay in the $n$th epoch. These power values are averaged over the duration of the corresponding epoch in the sense that the source and relay nodes actually transmit at constant powers $\frac{p_{s, n}}{\Delta_{n}}$ and $\frac{p_{r, n}}{1-\Delta_{n}}$ for $\Delta_{n}$ and $1-\Delta_{n}$ fractions of the epoch, respectively. For a relay with a fixed energy budget, an additional constraint is required to assure that the relay does not expend more energy than $E$, which we can express as $\sum_{n=1}^{N} l_{n} p_{r, n} \leq E$.

Lemma 2: For each feasible policy, there exists an equally well or better performing feasible policy in which the relay buffer is empty at the end of every epoch.

Proof: Suppose there exists a feasible policy in which the relay buffer is not empty at the end of the first epoch. Then there exists $\delta>0$ such that, if we swap a $\delta$-long portion of the first source subepoch with a $\delta$-long portion of some relay sub-epoch in the future, the relay empties its buffer at the end of the first epoch. This can be done without violating data causality at the relay because we know that in the original policy, the relay has to transmit the amount of data in its buffer at $s_{2}$ at a later time. Energy causality at the source is also preserved since the new policy only delays source transmission and the source has an infinite-capacity battery. One concern with the new policy is that power levels may no longer stay constant within an epoch, e.g., the relay may be transmitting with a larger power during the second $\delta$-long portion. However, since the rate in each hop is concave in power, two different power levels at any node can be equalized without changing the energy spent, and this last step may even result in a larger throughput. Repeating this process for epochs $2, \ldots, N-1$ concludes the proof.

As a corollary to Lemma 2, we can deduce that the optimal solutions of (2) and its fixed energy relay counterpart require that the relay buffer be empty at $s_{n}$ for all $n=2,3, \ldots, N+1$. In other words, the source and the relay must transmit the same amount of 
data in each epoch, i.e.,

$$
\tilde{C}\left(h_{1} p_{s, n}, \Delta_{n}\right)=\tilde{C}\left(h_{2} p_{r, n}, 1-\Delta_{n}\right), n=1,2, \ldots, N,
$$

where $\tilde{C}(x, y)=\frac{y}{2} \log (1+x / y)$. (3) plays an important role in our characterization of the optimal policy for (1).

\section{B. Optimal Policy with Constant Power Relay}

When the relay maintains constant power $P$, whenever it transmits, it will have a rate $R \triangleq C\left(h_{2} P\right)$. Consequently, (3) takes a simpler form as

$$
\tilde{C}\left(h_{1} p_{s, n}, \Delta_{n}\right)=\left(1-\Delta_{n}\right) R, n=1,2, \ldots, N .
$$

We define function $F:(0,1) \rightarrow(0, \infty)$ given by $F(\Delta)=$ $\frac{\Delta}{h_{1}}\left(2^{\frac{1-\Delta}{\Delta}} 2 R-1\right) . F$ is a continuous bijection whose domain $(0,1)$ is an open subset of $\mathbb{R}$, hence, by the invariance of domain theorem, $F$ is a homeomorphism, i.e., its inverse $F^{-1}$ is continuous. Note that $F$ maps any $\Delta_{n} \in(0,1)$ to a $p_{s, n}$ that, together with $\Delta_{n}$, satisfies (4). But $F^{-1}$ is also a continuous bijection, therefore it can be used to map any $p_{s, n} \in(0, \infty)$ to a $\Delta_{n}$ such that (4) is satisfied. We may also have $p_{s, n}=0$ which is not covered by $F^{-1}$; however it is obvious by inspection that in order to satisfy (4) with $p_{s, n}=0$, we must have $\Delta_{n}=1$. Then, as an extension of $F^{-1}$, we define $f:[0, \infty) \rightarrow(0,1]$ as $f(p)=F^{-1}(p)$ for $p \in(0, \infty)$ and $f(0)=1 . f$ is a continuous bijection since $\lim _{p \rightarrow 0} F^{-1}(p)=1$. Then, $f$ can be used to map any source power $p_{s, n}$ to a fraction $\Delta_{n}$ such that (4) is satisfied. Also, the continuity of $f$ allows us to use the bisection method as $f$ does not have a closed-form expression. Therefore, we can first solve optimization problem (2) to find the optimal $p_{s, n}$ values, and then use $f$ to find the appropriate $\Delta_{n}$ values for each epoch. In order to do this, we define the effective rate $g(p) \triangleq \tilde{C}\left(h_{1} p, f(p)\right)=(1-f(p)) R$. The reason we refer to $g$ as the effective rate is that the amount of data transmitted from the source to the destination in the $n$th epoch is exactly $l_{n} g\left(p_{s, n}\right)$ where $p_{s, n}$ is the average source power in the $n$th epoch.

The effective rate $g$ has some interesting properties that will lead us to the optimal policy. First of all, it is clear that $g(0)=0$. Also $g(p)$ is monotonically increasing in $p$. This follows from the fact that $f$ is a real-valued continuous bijection defined on an interval, hence it is monotone. Since $f(0)=1$ and $\lim _{p \rightarrow \infty} f(p)=0$, $f$ is monotonically decreasing in $p$ and $g(p)=(1-f(p)) R$ is monotonically increasing in $p$.

Lemma 3: $g(p)$ is concave in $p$.

Proof: Let $p_{1}$ and $p_{2}$ be two power values, and let $\Delta_{i}=$ $f\left(p_{i}\right), i=1,2$. Also let $\alpha \in[0,1]$ and define $\bar{\alpha}=1-\alpha$, $p=\alpha p_{1}+\bar{\alpha} p_{2}$ and $\Delta=\alpha \Delta_{1}+\bar{\alpha} \Delta_{2}$. Then,

$$
r(\alpha) \triangleq \alpha \tilde{C}\left(p_{1}, \Delta_{1}\right)+\bar{\alpha} \tilde{C}\left(p_{2}, \Delta_{2}\right) \leq \tilde{C}(p, \Delta),
$$

due to the log-sum inequality. Also, from the definition of $f$,

$$
r(\alpha)=\alpha\left(1-\Delta_{1}\right) R+\bar{\alpha}\left(1-\Delta_{2}\right) R=(1-\Delta) R .
$$

Then, we have $\tilde{C}(p, \Delta) \geq(1-\Delta) R$ and there exists $\tilde{\Delta} \leq \Delta$ such that this inequality is satisfied with equality if we replace $\Delta$ by $\tilde{\Delta}$. But, the new rate $(1-\tilde{\Delta}) R$ achieved by a convex combination of $p_{1}$ and $p_{2}$ is at least as large as $(1-\Delta) R$. Therefore, $g(p)$ is concave in $p$.

The properties of the effective rate given above make it possible to reduce our system model to a single-user energy harvesting channel where the achievable rate is given by $g(p)$ where $p$ is the instantaneous power. Then, the optimal source power values, $p_{s, n}$ can be found using the results of [1] where the optimal power policy for a single-user channel is proved to be the tightest string between the beginning and the end of the energy tunnel of the source. After finding $p_{s, n}$ for all $n$, the optimal fraction values can be found as $\Delta_{n}=f\left(p_{s, n}\right)$. Since $p_{s, n}$ and $\Delta_{n}=f\left(p_{s, n}\right)$ completely characterize the optimal solution, the solution with constant relay power is complete.

\section{Optimal Policy with Single Energy Arrival at the Relay}

When the relay has only one energy arrival, Lemma 2 still holds, implying that we can concentrate on finding an optimal policy in which the data buffer at the relay is empty at the end of each epoch. Hence, (3) must be satisfied for all $n=1,2, \ldots, N$, i.e., the source and the relay must transmit the same amount of data in any epoch. Similarly to the previous subsection, we can define the effective rate $\tilde{g}\left(p_{s, n}\right)$ as the left-hand side of (3).

Lemma 4: $\tilde{g}$ is concave in the average source power $p_{s, n}$.

Proof: Suppose we have an epoch of length $l$ and two policies for transmission in this epoch. The source and the relay allocate $E_{1}^{s}$ and $E_{1}^{r}$ units of energy, respectively in the first policy, and similarly $E_{2}^{s}$ and $E_{2}^{r}$ units of energy in the second policy. Define the average source power values as $p_{s, 1}=E_{1}^{s} / l$ and $p_{s, 2}=E_{2}^{s} / l$. Let $\Delta_{1}$ and $\Delta_{2}$ be the appropriate fraction values that satisfy (3). Let $\alpha \in[0,1]$ be arbitrary. Define $\bar{\alpha}=1-\alpha, p_{s}=\alpha p_{s, 1}+\bar{\alpha} p_{s, 2}, \bar{\Delta}_{i}=1-\Delta_{i}, i=1,2$, and $\Delta=\alpha \Delta_{1}+\bar{\alpha} \Delta_{2}$. Then,

$$
\tilde{r}(\alpha) \triangleq \alpha \tilde{C}\left(p_{s, 1}, \Delta_{1}\right)+\bar{\alpha} \tilde{C}\left(p_{s, 2}, \Delta_{2}\right) \leq \tilde{C}\left(p_{s}, \Delta\right),
$$

due to the log-sum inequality. From (3), we also have that

$$
\begin{aligned}
\tilde{r}(\alpha) & =\alpha \tilde{C}\left(E_{1}^{r} / l, \bar{\Delta}_{1}\right)+\bar{\alpha} \tilde{C}\left(E_{2}^{r} / l, \bar{\Delta}_{2}\right) \\
& \leq \tilde{C}\left(\left(\alpha E_{1}^{r}+\bar{\alpha} E_{2}^{r}\right) / l, 1-\Delta\right) .
\end{aligned}
$$

Thus, by operating at a convex combination of source power values $p_{s, 1}$ and $p_{s, 2}$, both sides of (3) are improved while keeping the energy spent by the relay constant at $\alpha E_{1}^{r}+\bar{\alpha} E_{2}^{r}$. While $\Delta$ may have to be adjusted to balance the two sides of (3), the new rate achieved by the convex combination of the two policies will never be lower than the convex combination of the rates achieved by the two policies individually.

It can similarly be argued that the effective rate $\tilde{g}\left(p_{s, n}\right)$ is monotonically increasing in $p_{s, n}$ and $\tilde{g}(0)=0$. Then, we can again consider our model as a single-user channel and find the optimal source power values $p_{s, n}$ using the results of [1]. Knowing the average source power for each epoch, we are left to determine the relay power values and appropriate fraction values for each epoch. Similarly to the previous subsection, we can define a function $\tilde{f}\left(p_{r, n} ; p_{s, n}\right)$ that is parameterized by the average source power $p_{s, n}$ and maps the average relay power $p_{r, n}$ to the appropriate fraction $\Delta_{n}$ such that (3) is satisfied. It can be shown that $\tilde{f}$ is a continuous bijection; thus the optimal $p_{r, n}$ values can be found by solving

$$
\begin{aligned}
\max _{p_{r, n}} & \sum_{n=1}^{N} l_{n} \frac{1-\tilde{f}\left(p_{r, n} ; p_{s, n}\right)}{2} \log \left(1+h_{2} \frac{p_{r, n}}{1-\tilde{f}\left(p_{r, n} ; p_{s, n}\right)}\right) \\
\text { s.t. } & \sum_{n=1}^{N} l_{n} p_{r, n} \leq E, p_{r, n} \geq 0, n=1,2, \ldots, N .
\end{aligned}
$$

The solution of (9) can be found readily by writing the KarushKuhn-Tucker (KKT) conditions, and is omitted due to limited space. Having found the relay power values $p_{r, n}$ for each epoch, the function $\tilde{f}$ can be used to find the optimal fraction values $\Delta_{n}$, and hence the optimal policy since it is completely characterized by $p_{s, n}, p_{r, n}$, and $\Delta_{n}, n=1,2, \ldots, N$. 


\section{Optimal Policies with Buffer Size Constraints}

We have so far found the optimal policy with $B_{\max }=\infty$ and shown that it can be replaced with another policy where the relay buffer is empty at the end of each epoch. This way, the required $B_{\max }$ is decreased to the maximum of the throughput values for each epoch. We can further claim that the relay does not have to use its buffer ever: Instead of pushing all relay transmission to the end of epochs, the relay can forward messages as soon as it receives them. This way, the source transmission of one symbol is immediately followed by a relay transmission of the same symbol. The source and relay power still remains constant within every epoch and the transmission time of the source and relay in an epoch is the same; therefore the throughput achieved remains unchanged. Energy causality at the source node is not violated because the source uses the same amount of energy and shifts its transmission towards the end of the epoch. The relay also spends the same amount of energy, but it has to shift its transmission back in time. This does not violate feasibility since, in our model, the relay either has constant transmission power or only one energy harvest. Thus, the optimal policies found in the previous two subsections are optimal with any $B_{\max }$, except the source and relay sub-epochs in an epoch has to be interleaved into each other such that the relay forwards symbols as soon as it receives them.

\section{NumericAl Results}

In this section, we present simulation results to assess our solution. Fig. 2 shows the average rate achieved by the optimal policy, along with upper and lower bounds where $P=2, T=1, h_{1}=h_{2}=1$. The average rate is computed for peak harvest rates for the source node varying from 0.1 to 2 . The upper bound is found by assuming that the source receives the same total amount of energy, but all at the beginning of transmission. This way, the energy causality constraints at the source are removed and since the source node is assumed to have an infinite-capacity battery, the set of feasible policies is enlarged. Therefore, the assumption of a non-energy harvesting source yields an upper bound on the average rate achieved. The lower bound is the case where the source does not have a battery to store energy for future use. This new constraint significantly simplifies the problem as the average source power for every epoch is immediately available and does not require solution of an optimization problem. But the new constraint also shrinks the feasible set; hence we get a lower bound on the achievable average rate. As can be seen, the average rate is concave and monotonically increasing in the peak harvest rate for the source node. The average rates achieved by the optimal policy are significantly higher than the lower bound. We also observe that the average rate curve becomes flatter as the peak harvest rate increases. This is due to the fact that, although larger energy harvests at the source increases the average rate, the relay rate is constant at $R=\frac{1}{2} \log \left(1+h_{2} P\right)$ and it limits the end-to-end rate achieved in each epoch.

\section{Discussion AND CONCLUSION}

In this paper we studied a two-hop channel with an energy harvesting source in two cases: first with a constant power relay and also with a relay that has one energy arrival (harvest). We found the optimal solutions for these two cases with the assumption that the relay has an infinite-capacity data buffer. We then showed that these optimal solutions do not actually require a data buffer at the relay. We finally provided numerical results to observe the performance of the optimal policy against upper and lower bounds.

With an energy harvesting relay with multiple energy arrivals, the buffer size may affect the optimal policy and Lemma 2 no longer

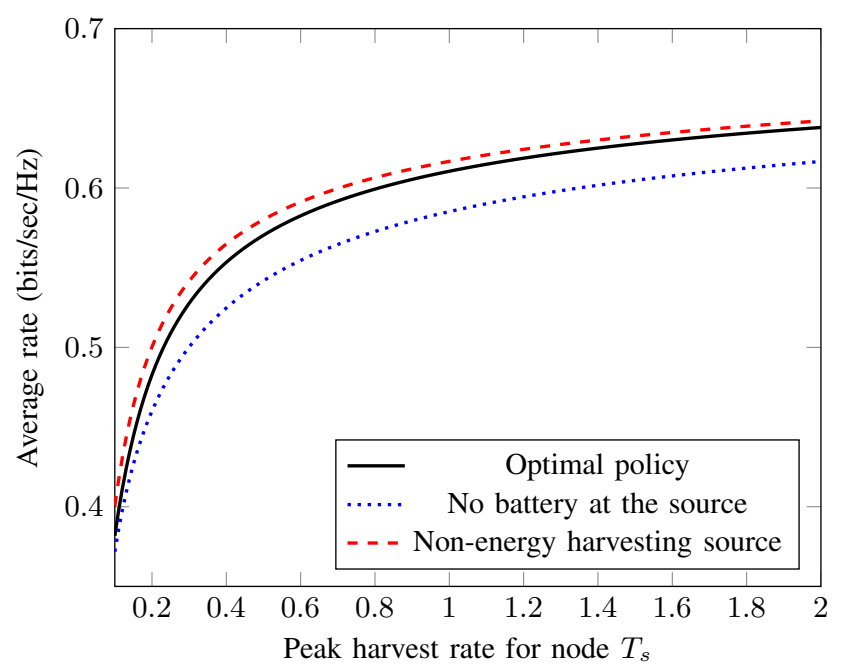

Fig. 2: Performance comparison of the optimal policy.

holds. As an example, suppose there are two arrivals at the relay: one at $s_{1}$ with little harvested energy and another one just before the deadline $T$, with an amount of energy sufficient to transmit all the data in the relay buffer by $T$. Since the relay receives most of its energy near $T$, the relay would need to store data in its buffer until the second arrival, and then transmit it before the deadline for maximum throughput.

\section{REFERENCES}

[1] J. Yang and S. Ulukus, "Optimal packet scheduling in an energy harvesting communication system," Communications, IEEE Transactions on, vol. 60, no. 1, pp. 220-230, 2012.

[2] K. Tutuncuoglu and A. Yener, "Optimum transmission policies for battery limited energy harvesting nodes," Wireless Communications, IEEE Transactions on, vol. 11, no. 3, pp. 1180-1189, 2012.

[3] O. Ozel, K. Tutuncuoglu, J. Yang, S. Ulukus, and A. Yener, "Transmission with energy harvesting nodes in fading wireless channels: Optimal policies," IEEE Journal on Selected Areas in Communications, vol. 29, no. 8, pp. $1732-1743$, Sep. 2011.

[4] J. Yang and S. Ulukus, "Optimal packet scheduling in a multiple access channel with energy harvesting transmitters," Communications and Networks, Journal of, vol. 14, no. 2, pp. 140-150, 2012.

[5] J. Yang, O. Ozel, and S. Ulukus, "Broadcasting with an energy harvesting rechargeable transmitter," Wireless Communications, IEEE Transactions on, vol. 11, no. 2, pp. 571-583, 2012.

[6] M. Antepli, E. Uysal-Biyikoglu, and H. Erkal, "Optimal packet scheduling on an energy harvesting broadcast link," Selected Areas in Communications, IEEE Journal on, vol. 29, no. 8, pp. 1721-1731, 2011.

[7] K. Tutuncuoglu and A. Yener, "Sum-rate optimal power policies for energy harvesting transmitters in an interference channel," Communications and Networks, Journal of, vol. 14, no. 2, pp. $151-161,2012$.

[8] C. Huang, R. Zhang, and S. Cui, "Throughput maximization for the Gaussian relay channel with energy harvesting constraints," arXiv preprint arXiv:1109.0724, 2011.

[9] D. Gunduz and B. Devillers, "Two-hop communication with energy harvesting," in Computational Advances in Multi-Sensor Adaptive Processing (CAMSAP), 2011 4th IEEE International Workshop on. IEEE, 2011, pp. 201-204.

[10] O. Orhan and E. Erkip, "Optimal transmission policies for energy harvesting two-hop networks," in Information Sciences and Systems (CISS), 2012 46th Annual Conference on. IEEE, 2012, pp. 1-6.

[11] _ , "Energy harvesting two-hop networks: Optimal policies for the multi-energy arrival case," in Sarnoff Symposium (SARNOFF), 2012 35th IEEE. IEEE, 2012, pp. 1-6.

[12] Y. Luo, J. Zhang, and K. Letaief, "Optimal scheduling and power allocation for two-hop energy harvesting communication systems," arXiv preprint arXiv:1212.5394, 2012. 